\title{
Avaliação da ocorrência do evento adverso flebite em pacientes de uma Unidade de Internação Clínica*
}

\section{Evaluation of phlebitis adverse event occurrence in patients of a Clinical Inpatient Unit Evaluación del acaecimiento del evento adverso flebitis en pacientes de una Unidad de Hospitalización Clínica}

Como citar este artigo:

Furlan MS, Lima AFC. Evaluation of phlebitis adverse event occurrence in patients of a Clinical Inpatient Unit. Rev Esc Enferm USP. 2021;55:e03755. https:// doi.org/10.1590/S1980-220X2020017103755

\section{Maryana da Silva Furlan ${ }^{1}$}

Antônio Fernandes Costa Lima ${ }^{2}$

* Extraído da dissertação: "Custo direto da ocorrência do evento adverso flebite em uma Unidade de Internação Clínica”, Escola de Enfermagem, Universidade de São Paulo, 2019.

${ }^{1}$ Universidade de São Paulo, Escola de Enfermagem, Programa de Pós-Graduação em Enfermagem, São Paulo, SP, Brasil.

${ }^{2}$ Universidade de São Paulo, Escola de Enfermagem, Departamento de Orientação Profissional, São Paulo, SP, Brasil.

\begin{abstract}
Objective: To analyze the causes and reasons associated with the occurrence of phlebitis in a Inpatient Medical Unit in a large and private general hospital. Method: Quantitative, exploratory-descriptive, retrospective and documentary research, carried out by consulting the electronic forms of notification of the occurrence of phlebitis in 2017. Results: A total of 107 phlebitis related to 96 patients were reported, most of them (91.7\%) with phlebitis, being male (53.1\%), aged 60-69 years old (23.0\%) and with a hospital stay of less than four days (30.2\%). Most (68.2\%) of the notifications were made by nurses, with the occurrence of phlebitis predominating in devices with less than $24 \mathrm{~h}$ (38.3\%); with the classification of phlebitis grade 2 (45.8\%); with antibiotics infusion (46.7\%); with the location of the bed far from the nursing station $(52.3 \%)$; and with the presence of a companion (82.2\%). The damage classification indicated that $93.5 \%$ of the patients suffered mild damage, $4.7 \%$ moderate damage and $1.9 \%$ did not suffer any damage. Conclusion: Knowing the causes and reasons associated with the occurrence of phlebitis can support decision-making, management and care processes regarding investments in preventive or risk mitigation strategies.
\end{abstract}

DESCRIPTORS

Inpatients; Catheterization, Peripheral; Phlebitis; Nursing Care; Hospital Units. 


\section{INTRODUÇÃO}

Em algum momento, a terapia intravenosa (TIV) é indicada durante a hospitalização para aproximadamente $80 \%$ dos pacientes. $\mathrm{E}$ desses, de $20 \%$ a $70 \%$ desenvolvem o evento adverso (EA) flebite ${ }^{(1)}$, cujos sinais e sintomas correspondem a edema, calor local, hiperemia, cordão fibroso no trajeto do vaso, dor e, ainda, podendo haver presença de secreção purulenta em sítio de inserção do cateter ${ }^{(2)}$.

A flebite pode ser mecânica, química, bacteriana ou pós-infusional, sendo classificada em: grau 1, presença de hiperemia no sítio de inserção do cateter, com ou sem dor local; grau 2, dor no sítio de inserção do cateter com hiperemia e/ou edema e endurecimento; grau 3, dor no sítio de inserção do cateter com hiperemia, endurecimento e formação de cordão venoso palpável; grau 4, dor no sítio de inserção do cateter com hiperemia, endurecimento e formação de cordão venoso palpável com drenagem purulenta ${ }^{(2)}$.

Estudo realizado em uma unidade de internação clínica (UIC) identificou a presença de flebite em $60 \%$ dos pacientes e em 55,6\% dos acessos venosos periféricos (AVP), cuja ocorrência foi associada ao aumento do tempo de permanência desses pacientes ${ }^{(3)}$. Outro estudo evidenciou que pacientes hospitalizados em unidades cirúrgicas e ortopédicas possuíam risco menor de apresentar flebite quando comparados aos que estavam em UIC que, por sua vez, apresentaram período de internação prolongado, bem como o uso de corticosteroides e antimicrobianos, fatores relacionados ao desenvolvimento de flebite ${ }^{(4)}$.

O livro To err is human mostrou o impacto global da segurança do paciente e o número de mortes decorrentes das falhas nos cuidados à saúde ${ }^{(5)}$. Em 2004, a World Alliance for Patient Safety ${ }^{(6)}$ reconheceu a magnitude do problema e objetivou identificar prioridades na área da segurança do paciente, além de estabelecer medidas para reduzir os riscos e a ocorrência de $\mathrm{EA}^{(7)}$. No Brasil, a Portaria no 529 , de 1o de abril de 2013, do Ministério da Saúde, instituiu o Programa Nacional de Segurança do Paciente (PNSP), que tem como premissa contribuir para a assistência à saúde de qualidade em todos os níveis de atenção à saúde no país ${ }^{(8)}$. Reconhecendo os danos causados aos pacientes, decorrentes da assistência a saúde, na 72a Assembleia Mundial de Saúde, em 2019, a World Health Organization (WHO) adotou a resolução WHA72.6, a respeito da ação global sobre a segurança do paciente e reconheceu esse tema como uma prioridade de saúde global ${ }^{(9)}$.

A flebite é um EA evitável e há estratégias que podem ser implementadas, isso a partir do conhecimento dos fatores de risco para prevenir e/ou minimizar a sua ocorrência, concorrendo para a prestação de cuidados livre de danos. Um estudo de coorte, com 171 pacientes com AVP, investigou a associação de fatores de risco e a ocorrência de flebites durante o uso e após a retirada do AVP, $51,5 \%$ dos pacientes eram homens, com tempo de permanência do cateter até 72 horas e $58,2 \%$ dos medicamentos utilizados eram sedativos/ analgésicos. Identificou a necessidade do conhecimento a respeito dos fatores de risco para flebite, a fim de contribuir no planejamento do cuidado pelo enfermeiro e na identificação dos pacientes que podem desenvolver flebite ${ }^{(10)}$.

Os profissionais de saúde, notadamente os de enfermagem, precisam compreender a importância da sua participação qualificada, isso conforme seu nível de governabilidade e em relação à adoção de medidas preventivas do EA flebite, visando à obtenção de resultados favoráveis aos pacientes/ usuários, familiares e a si próprios, para a organização e para a sociedade. Entretanto, para direcionar a escolha das estratégias de prevenção é preciso conhecer as causas e consequências desse EA, com essas informações será possível melhorar os processos assistenciais e gerenciais de forma contínua, e assegurar a prestação de serviços de saúde livre de danos e com redução dos riscos. Nessa perspectiva, este estudo objetiva analisar quais as causas e os motivos associados à ocorrência de flebite na UIC de um hospital geral, de grande porte e privado.

\section{MÉTODO}

\section{TIPO DO ESTUDO}

Trata-se de pesquisa quantitativa, exploratório-descritiva, retrospectiva e documental.

\section{Cenário}

O Hospital Geral, campo de estudo, é privado, localiza-se na cidade de São Paulo e possui 512 leitos de internação, sendo 38 leitos destinados à UIC. Compuseram o presente estudo as notificações da ocorrência do EA flebite em pacientes da UIC, realizadas por profissionais de saúde por meio do preenchimento do formulário eletrônico disponível na intranet do Hospital.

\section{CRitérios de SeleÇão}

Foram selecionados todos os formulários eletrônicos relativos à notificação da ocorrência de flebite em pacientes da UIC, no período de janeiro a dezembro de 2017.

\section{Coleta de dados}

A partir da estruturação dos formulários eletrônicos para notificação de flebite no hospital, foi criado um instrumento para documentar as seguintes informações: autor (enfermeiro, técnico de enfermagem, anônimo, outro) e data da notificação; número de atendimento do paciente; data do atendimento; nome do médico; número de atendimento; idade, sexo e leito do paciente; período (manhã, tarde, noite); grau da flebite (grau 1: hiperemia, grau 2: hiperemia + dor local, grau 3: hiperemia + dor local + cordão palpável, grau 4: hiperemia + dor local + cordão palpável e drenagem purulenta); nome do fármaco envolvido; tempo de permanência do dispositivo intravenoso; classificação do dano (grave, moderado, leve, nenhum); avaliação risco para o desenvolvimento de flebite (alto, moderado, baixo, sem risco); informações adicionais (como exemplos, print da prescrição, fotografia do local). Os dados constantes dos instrumentos foram inseridos em planilhas eletrônicas. 


\section{ANÁLISE E TRATAMENTO DOS DADOS}

Os dados contínuos e semicontínuos foram inicialmente comparados com a curva de Gauss por meio do teste de distância K-S (Kolmogorov-Smirnov), determinados como não paramétricos e expressos por intermédio de mediana, percentis e comparados pelo teste de KrusKal-Wallis, com pós-teste de Muller-Dunn na comparação de três ou mais grupos.

Os dados categóricos foram representados por frequência absoluta (n) e relativa (\%), sendo as matrizes de contingência analisadas pelo teste de Qui-quadrado de Pearson ou Teste Exato de Fisher, quando necessário. As matrizes complexas foram particionadas em matrizes simples para melhor determinação da causalidade. Foi considerado, para todo o estudo, risco $\alpha \leq 0,05$ de cometer erro tipo I ou de $1^{\text {a }}$ espécie, além de risco $\beta \leq 0,20$ de cometer erro tipo II ou de $2^{\mathrm{a}}$ espécie.

\section{Aspectos éticos}

A pesquisa foi aprovada pelo Comitê de Ética em Pesquisa do hospital, por meio do parecer consubstanciado número: 2.500.262, de 19 de fevereiro de 2018, atendendo todas as especificações da Resolução n ${ }^{\circ} 466$, de 12 de dezembro de 2012, que aprova as diretrizes e normas regulamentadoras de pesquisas envolvendo seres humanos.

\section{RESULTADOS}

Em 2017 ocorreram 3.028 internações na UIC, sendo notificadas 107 flebites associadas a 96 pacientes, a maioria (91,7\%) acometida por uma flebite. A partir das notificações analisadas verificou-se, conforme apresentado na Tabela 1 , que a maioria $(53,1 \%)$ dos pacientes era do sexo masculino, na faixa etária de 60-69 anos (23,0\%) e com tempo de permanência inferior a quatro dias $(30,2 \%)$.

Tabela 1 - Distribuição dos pacientes acometidos por flebite na Unidade de Internação Clínica, conforme sexo, faixa etária e tempo de permanência. São Paulo, SP, Brasil, 2019.

\begin{tabular}{lcc}
\hline Variáveis & N & $\%$ \\
\hline Sexo & 45 & 46,9 \\
$\quad$ Feminino & 51 & 53,1 \\
$\quad$ Masculino & 1 & \\
\hline Faixa etária (em anos) & 3 & 1,0 \\
10-19 & 15 & 3,1 \\
$20-29$ & 15 & 15,6 \\
$30-39$ & 13 & 15,6 \\
$40-49$ & 22 & 13,5 \\
$50-59$ & 13 & 23,0 \\
$60-69$ & 10 & 13,5 \\
$70-79$ & 4 & 10,4 \\
$80-89$ & & 4,1 \\
$90-99$ & 29 & 30,2 \\
\hline Tempo de permanência (dias) & 25 & 26,0 \\
$<4$ dias & 25 & 26,0 \\
$5-7$ dias & 17 & 17,8 \\
$8-11$ dias & $\mathbf{9 6}$ & $\mathbf{1 0 0 , 0}$ \\
\hline 12 dias & &
\end{tabular}

As hipóteses diagnósticas médicas dos 96 pacientes foram agrupadas em categorias, a maioria, $70,8 \%$, estava internada por afecções infecciosas; seguida por $16,6 \%$ por afecções do sistema nervoso central e periférico; $9,4 \%$ por afecções do aparelho digestivo. Entre as doenças pré-existentes destacaram-se: hipertensão arterial sistêmica - HAS $(22,9 \%)$, diabetes mellitus - DM $(15,6 \%)$ e dislipidemia (12,5\%). Vale ressaltar que $19,8 \%$ dos pacientes apresentavam mais do que uma doença pré-existente.

Janeiro (13,1\%), junho (12,1\%), julho (10,3\%) e outubro $(10,3 \%)$ foram os meses com o maior número de notificações. Os turnos da tarde e da manhã apresentaram o maior quantitativo de notificações (40,2\% e 38,3\%), realizadas por enfermeiros (68,2\%); 29,9\% das notificações foram anônimas; $1,9 \%$ realizadas por técnicos de enfermagem. Não foi constatada diferença estatística significante em relação à distribuição do grau das 107 flebites e às variáveis sexo, faixa etária e tempo de permanência dos pacientes.

Segundo a Tabela 2, a maioria (52,3\%) das flebites ocorreu em pacientes que utilizavam o dispositivo intravenoso Jelco ${ }^{\circledR}$, com tempo de permanência do dispositivo de $24 \mathrm{~h}$ (38,3\%), com prevalência da flebite grau $2(45,8 \%)$, recebendo antibióticos (46,7\%), com os leitos localizados distantes do posto de enfermagem $(52,3 \%)$ e com a presença de acompanhante $(82,2 \%)$.

Tabela 2 - Caracterização das flebites notificadas quanto ao tipo de dispositivo, tempo de permanência do dispositivo, grau da flebite, categoria do fármaco infundido - São Paulo, SP, Brasil, 2019.

\begin{tabular}{|c|c|c|}
\hline Variáveis & $\mathbf{N}$ & $\%$ \\
\hline \multicolumn{3}{|l|}{ Tipo de dispositivo } \\
\hline Jelco ${ }$ & 56 & 52,3 \\
\hline Intima $\mathbb{R}$ & 51 & 47,7 \\
\hline \multicolumn{3}{|c|}{ Tempo de permanência do dispositivo } \\
\hline $24 \mathrm{~h}$ & 41 & 38,3 \\
\hline $48 \mathrm{~h}$ & 28 & 26,1 \\
\hline $72 \mathrm{~h}$ & 18 & 16,8 \\
\hline $96 \mathrm{~h}$ & 6 & 5,6 \\
\hline $120 \mathrm{~h}$ & 6 & 5,6 \\
\hline $144 \mathrm{~h}$ & 5 & 4,8 \\
\hline $168 \mathrm{~h}$ & 2 & 1,9 \\
\hline Pós-retirada & 1 & 0,9 \\
\hline \multicolumn{3}{|l|}{ Grau da flebite* } \\
\hline Grau 1 & 32 & 29,9 \\
\hline Grau 2 & 49 & 45,8 \\
\hline Grau 3 & 24 & 22,4 \\
\hline Grau 4 & 2 & 1,9 \\
\hline \multicolumn{3}{|l|}{ Categoria do fármaco } \\
\hline Analgésico & 9 & 8,4 \\
\hline Anti-histamínico & 1 & 0,9 \\
\hline Anti-inflamatório & 2 & 1,9 \\
\hline Antianêmico & 1 & 0,9 \\
\hline Antiemético & 3 & 2,8 \\
\hline Antifúngico & 3 & 2,8 \\
\hline Antibiótico & 50 & 46,7 \\
\hline Antiviral & 7 & 6,5 \\
\hline Cristaloide & 1 & 0,9 \\
\hline Diurético & 1 & 0,9 \\
\hline
\end{tabular}




\begin{tabular}{lcc}
...continuação & N & $\%$ \\
\hline Variáveis & 10 & 9,3 \\
\hline Imunoglobulina & 15 & 14 \\
Solução eletrolítica & 4 & 3,7 \\
$\quad$ Ausência de registro & & \\
Localização do leito & 51 & 47,7 \\
$\quad$ Próximo ao posto de enfermagem & 56 & 52,3 \\
$\quad$ Distante do posto de enfermagem & & \\
Presença de acompanhante & 88 & 82,2 \\
Sim & 19 & 17,8 \\
$\quad$ Não &
\end{tabular}

$\mathrm{N}=107$
Analisando na Tabela 3 a relação entre as doenças pré-existentes e o grau de flebite, houve diferença estatística significante da ocorrência de flebite grau 2 associada às doenças pré-existentes HAS $(\mathrm{p}=0,020)$, transtornos mentais, comportamentais $(\mathrm{p}=0,020)$ e neuropatia $(\mathrm{p}=0,005)$. A maioria $(40,0 \%)$ dos pacientes com HAS apresentaram flebite grau $2(15-68,2 \%)$ e grau $3(6-27,3 \%)$, além disso três $(75,0 \%)$ pacientes com insuficiência renal crônica e nove $(75,0 \%)$ com dislipidemia também apresentaram flebite grau 2. O DM esteve associado ao maior quantitativo de pacientes com flebite grau $2(53,3 \%)$ e grau $3(26,7 \%)$, ademais três pacientes $(60,0 \%)$ com hipótese diagnóstica de sepse apresentaram flebite grau 3.

Tabela 3 - Distribuição do grau das flebites notificadas segundo as variáveis doenças pré-existentes e hipóteses diagnósticas - São Paulo, SP, Brasil, 2019.

\begin{tabular}{|c|c|c|c|c|c|c|c|c|c|c|}
\hline \multirow{2}{*}{$\begin{array}{l}\text { Grau de Flebite* } \\
\text { Variáveis }\end{array}$} & & \multicolumn{2}{|c|}{ Grau 1} & \multicolumn{2}{|c|}{ Grau 2} & \multicolumn{2}{|c|}{ Grau 3} & \multicolumn{2}{|c|}{ Grau 4} & \multirow{2}{*}{$\frac{\chi^{2} \text { de Pearson** }}{\text { Sig. }}$} \\
\hline & & $\mathbf{N}$ & $\%$ & $\mathbf{N}$ & $\%$ & $\mathrm{~N}$ & $\%$ & $\mathbf{N}$ & $\%$ & \\
\hline \multicolumn{11}{|l|}{ Doenças pré-existentes } \\
\hline \multirow{2}{*}{$\begin{array}{l}\text { Hipertensão Arterial } \\
\text { Sistêmica }\end{array}$} & Não & 31 & $36,5 \%$ & 34 & $40,0 \%$ & 18 & $21,2 \%$ & 2 & $2,4 \%$ & \multirow{2}{*}{0,02} \\
\hline & Sim & 1 & $4,5 \%$ & 15 & $68,2 \%$ & 6 & $27,3 \%$ & 0 & $0,0 \%$ & \\
\hline \multirow{2}{*}{$\begin{array}{l}\text { Transtornos mentais e } \\
\text { comportamentais }\end{array}$} & Não & 31 & $30,7 \%$ & 45 & $44,6 \%$ & 24 & $23,8 \%$ & 1 & $1,0 \%$ & \multirow[t]{2}{*}{0,02} \\
\hline & Sim & 1 & $16,7 \%$ & 4 & $66,7 \%$ & 0 & $0,0 \%$ & 1 & $16,7 \%$ & \\
\hline \multirow{2}{*}{ Neuropatia } & Não & 31 & $30,1 \%$ & 47 & $45,6 \%$ & 24 & $23,3 \%$ & 1 & $1,0 \%$ & \multirow{2}{*}{0,005} \\
\hline & Sim & 1 & $25,0 \%$ & 2 & $50,0 \%$ & 0 & $0,0 \%$ & 1 & $25,0 \%$ & \\
\hline \multirow{2}{*}{$\begin{array}{l}\text { Insuficiência Renal } \\
\text { Crônica }\end{array}$} & Não & 31 & $30,1 \%$ & 46 & $44,7 \%$ & 24 & $23,3 \%$ & 2 & $1,9 \%$ & \multirow{2}{*}{0,611} \\
\hline & Sim & 1 & $25,0 \%$ & 3 & $75,0 \%$ & 0 & $0,0 \%$ & 0 & $0,0 \%$ & \\
\hline \multirow{2}{*}{ Dislipidemia } & Não & 31 & $32,6 \%$ & 40 & $42,1 \%$ & 22 & $23,2 \%$ & 2 & $2,1 \%$ & \multirow{2}{*}{0,166} \\
\hline & Sim & 1 & $8,3 \%$ & 9 & $75,0 \%$ & 2 & $16,70 \%$ & 0 & $0,0 \%$ & \\
\hline \multirow{2}{*}{ Sepse } & Não & 31 & $30,4 \%$ & 48 & $47,1 \%$ & 21 & $20,6 \%$ & 2 & $2,0 \%$ & \multirow{2}{*}{0,228} \\
\hline & Sim & 1 & $20,0 \%$ & 1 & $20,0 \%$ & 3 & $60,0 \%$ & 0 & $0,0 \%$ & \\
\hline
\end{tabular}

$\mathrm{N}=107$

Quando comparada a variável função do relator com o grau da flebite, houve relação estatística significante $(p=0,007)$ entre a flebite grau 2 e a notificação realizada pelo enfermeiro em relação à notificação de forma anônima. Os técnicos de enfermagem notificaram apenas duas flebites referentes ao grau 1 . Dos $50(100,0 \%)$ pacientes que receberam antibiótico, $42,0 \%$ apresentaram flebite grau 2 e $28 \%$ flebite grau 3. Entre os $10(100,0 \%)$ pacientes que utilizaram imunoglobulina, 50,0\% apresentaram flebite grau 3. Quanto aos pacientes localizados distantes do posto de enfermagem, $28(50,0 \%)$ apresentaram flebite grau 2 , seguidos do grau 3 $(17,9 \%)$ e do grau $4(3,6 \%)$.

No hospital que foi campo de estudo, o enfermeiro classifica o risco para o desenvolvimento de flebite a cada 24 horas, como parte integrante da Sistematização da Assistência de Enfermagem. Em relação a essa classificação, $47,7 \%$ dos pacientes corresponderam como baixo risco, $23,4 \%$ como risco moderado, $22,4 \%$ como alto risco e $6,5 \%$ como sem risco. Não houve diferença estatística significante entre o risco para desenvolvimento de flebite e as variáveis: sexo, faixa etária, tempo de permanência dos pacientes, doenças pré-existentes, agrupamento das hipóteses diagnósticas médicas, mês da ocorrência, turno (manhã, tarde ou noite) e categoria profissional do relator.

No que diz respeito ao dano causado, $93,5 \%$ dos pacientes com flebite sofreram danos leve, $4,7 \%$, danos moderados e 1,9\% não sofreram danos. Não houve registro da ocorrência de danos graves ou óbito. Mas houve diferença estatística significante dos danos leves associados às doenças pré-existentes transtorno mental e de comportamento $(\mathrm{p}=$ $0,014)$ e hipotireoidismo $(p=0,042)$, com relação à hipótese diagnóstica médica de sepse $(\mathrm{p}=0,023)$.

Quanto ao dano causado pela flebite, não houve diferença estatística significante comparada as variáveis: sexo, faixa etária, tempo de permanência dos pacientes, mês da ocorrência, turno e categoria profissional do relator, tipo de dispositivo intravenoso, tempo de permanência do dispositivo, classe do fármaco, localização do leito em relação ao posto de enfermagem e presença de acompanhante. Considerando-se a associação entre classificação de danos 
e os fármacos infundidos, apenas a Oxacilina $(p=0,007)-$ pertencente à classe dos antibióticos - apresentou diferença estatística significante com o dano leve.

Na Tabela 4, constata-se que não houve diferença estatística significante correlacionando às variáveis: tempo de permanência do cateter com o grau da flebite, classificação dos danos e avaliação de risco. O tempo mediano de permanência do dispositivo na flebite grau 1 foi de 2,5 dias; na flebite grau 2, de três dias; na flebite grau 3, de três dias; na flebite grau 4, de seis dias. O tempo mediano de permanência do dispositivo nos pacientes que apresentaram dano moderado foi de três dias, com variação entre três e quatro dias.

Tabela 4 - Distribuição do tempo de permanência do cateter, segundo as variáveis grau de flebite, classificação do dano e avaliação de risco - São Paulo, SP, Brasil, 2019.

\begin{tabular}{|c|c|c|c|c|c|}
\hline \multirow{2}{*}{ Variáveis } & & \multicolumn{4}{|c|}{ Tempo de permanência (em dias) do cateter* } \\
\hline & & Mediana & $25 \%$ & $75 \%$ & Sig.** \\
\hline \multirow{4}{*}{ Grau de flebite } & Grau 1 & 2,5 & 2,0 & 4,0 & \multirow{4}{*}{0,173} \\
\hline & Grau 2 & 3,0 & 2,0 & 5,0 & \\
\hline & Grau 3 & 3,0 & 2,0 & 3,5 & \\
\hline & Grau 4 & 6,0 & 3,0 & 9,0 & \\
\hline \multirow{4}{*}{ Classificação do dano } & Nenhum & 2,0 & 1,0 & 3,0 & \multirow{4}{*}{0,81} \\
\hline & Leve & 3,0 & 2,0 & 4,0 & \\
\hline & Moderado & 3,0 & 3,0 & 4,0 & \\
\hline & Grave & - & - & - & \\
\hline \multirow{4}{*}{ Avaliação risco } & Sem risco & 2,0 & 1,0 & 3,0 & \multirow{4}{*}{0,324} \\
\hline & Baixo & 3,0 & 2,0 & 4,0 & \\
\hline & Moderado & 3,0 & 3,0 & 4,0 & \\
\hline & Alto & 3,0 & 2,0 & 4,0 & \\
\hline
\end{tabular}

*Dados expressos em mediana e percentis, ${ }^{* *}$ Teste de Kruskal-Wallis

\section{DISCUSSÃO}

Com frequência, os pacientes da UIC que permanecem hospitalizados por um longo período, geralmente, são idosos com alta dependência dos cuidados de enfermagem, necessitando, inclusive, de suporte da equipe de desospitalização para auxílio no processo de recuperação e alta hospitalar. Há também pacientes internados para tratamento de afecções infecciosas e neurológicas que permanecem um período prolongado. Além disso, a UIC é referência para pacientes da psiquiatria que demandam dias de hospitalização para o adequado controle de sua patologia.

Estudo realizado em um hospital do noroeste paulista evidenciou que os pacientes que tiveram flebite permaneceram internados em média 22,3 dias, 53,4\% desses pacientes por um período inferior a $15 \mathrm{dias}^{(11)}$. No Distrito Federal, o tempo médio de hospitalização de pacientes com flebite foi de 17,86 dias ( $\mathrm{DP} \pm 14,37$ ), variando de dois a $93 \operatorname{dias}^{(3)}$.

Neste estudo, a maioria $(53,1 \%)$ dos pacientes com flebite eram do sexo masculino, resultado semelhante aos encontrados na literatura ${ }^{(10-14)}$. Em pesquisa realizada na UIC de um hospital universitário, $51,5 \%$ dos pacientes com flebite eram homens ${ }^{(10)}$. O quantitativo de homens internados com doenças crônicas não transmissíveis e complicações decorrentes da falta de tratamento é maior, pois esses referem não valorizar sinais e sintomas apresentados e apenas buscam por tratamento hospitalar após semanas e meses sem melhora do quadro. Além disso, tendem a serem mais resistentes às orientações da equipe de saúde em relação ao autocuidado.

No Zimbábue, uma pesquisa avaliou os fatores de risco relacionados ao paciente e ao AVP para o desenvolvimento de flebite, e indicou que a idade dos pacientes variou de 23-80 anos, sendo que $28,2 \%$ dos pacientes tinham entre 30-39 anos, 26,0\% tinham entre $40-49$ anos e $23,9 \%$ tinham mais de 60 anos $^{(14)}$. Outra pesquisa mostrou que $53,0 \%$ dos pacientes com flebite possuíam idade superior a 60 anos $^{(2)}$, resultado próximo ao encontrado em outro estudo, no qual $43,7 \%$ das notificações de flebite estiveram associadas a pacientes com 60 anos ou mais ${ }^{(11)}$.

Os pacientes idosos possuem maior número de doenças pré-existentes que são fatores de risco para o desenvolvimento de flebite ${ }^{(15)}$. Este estudo evidenciou que os pacientes com flebite possuíam como doenças pré-existentes HAS, DM e dislipidemia, corroborando achados disponíveis na literatura ${ }^{(16-17)}$. O mesmo ocorreu com a hipótese diagnóstica, sendo a afecção infecciosa a causa principal de internação desses pacientes ${ }^{(16)}$. Já na Amazônia ocidental brasileira, a presença de doença crônica e a hipótese diagnóstica de infecção mostrou relação estatística significativa para a flebite em pacientes internados ${ }^{(18)}$. Múltiplas doenças pré-existentes também estiveram presentes em 89,3\% dos indivíduos que tiveram flebite no Zimbábue ${ }^{(14)}$.

No que se refere aos meses de maior notificação da flebite na UIC, ressalta-se que durante o inverno o quantitativo de pacientes internados com afecções respiratórias foi maior, 
demandando o uso de antibióticos que requereram a instalação e manutenção de AVP, fatores que poderiam contribuir para o desenvolvimento de flebite.

Estudo realizado em um Hospital Universitário avaliou os incidentes relacionados à segurança do paciente, destes, 344 eram EA, e a flebite foi a quarta causa de notificação com $13,8 \%$, já os enfermeiros foram responsáveis por $70,6 \%$ das notificações ${ }^{(19)}$. A notificação dos EA contribui para a melhoria do cuidado, possibilitando identificar as causas e propor melhorias para mitigar a sua ocorrência.

Quanto ao período de notificação da ocorrência de flebite, uma pesquisa evidenciou a prevalência no período da manhã (43,7\%), devido maior quantitativo de procedimentos e de profissionais de saúde para viabilizá-los, seguido pelo período da tarde $(40,7 \%)^{(19)}$. Um relatório divulgado pela Agência Nacional de Vigilância Sanitária (Anvisa) refere que, entre janeiro de 2014 e dezembro de 2018, 59,6\% das notificações de EA foram realizadas no período das 7 às $19 \mathrm{~h}$; no Estado de São Paulo esse valor correspondeu a 62,7\% das notificações. No período supracitado foram notificadas 14.428 flebites no Brasil ${ }^{(2))}$.

$\mathrm{Na}$ Turquia, uma pesquisa transversal determinou o efeito do tempo da troca do cateter venoso periférico no desenvolvimento de flebite. Os dispositivos inseridos por menos de $48 \mathrm{~h}$ tiveram 5,8 vezes mais chance de apresentar flebite, aqueles que estavam inseridos entre 49 a $96 \mathrm{~h}$ o risco era 2,8 vezes maior, ambos comparados àqueles que estavam com o cateter de 97-120h. A incidência de flebite foi maior nos AVP com 48h ou menos, seguido daqueles com 49-96h e 97 a $120 \mathrm{~h}^{(21)}$. No Vale do Paraíba, a média de permanência dos cateteres venosos nos pacientes que desenvolveram flebite foi de $49 h^{(12)}$. Estudo que analisou a incidência de flebite em uma UIC verificou que $36,2 \%$ dos AVP possuíam tempo de permanência de até $72 \mathrm{~h}$, e $63,8 \%$ dos AVP possuíam um tempo superior a $72 \mathrm{~h}^{(3)}$.

$\mathrm{Na}$ Austrália, uma pesquisa com 3.283 pacientes avaliou 5.907 AVP divididos em dois grupos: substituição do AVP, quando clinicamente indicado; e substituição do AVP de rotina, em ambos os grupos, nesses casos 7,0\% dos pacientes tiveram flebite. O estudo evidenciou que os dispositivos poderiam ser substituídos, quando clinicamente indicado, assim se evitaria a punção de até seis milhões de dispositivos, por conseguinte diminuir-se-iam os custos relacionados à mão de obra e uso de materiais para o procedimento ${ }^{(22)}$.

No Paraná, a flebite grau 2 correspondeu a maioria das flebites notificadas, seguido de grau $3^{(13)}$. Um hospital da Rede Sentinela, no município do Recife, mostrou que 63,2\% foram classificadas como grau 1, seguidas de $27,0 \%$ do grau 2 e de $5,3 \%$ do grau $3^{(23)}$. No Vale da Paraíba, $41,6 \%$ das notificações foram relacionados à flebite grau $1 ; 37,5 \%$ ao grau $2 ; 16,7 \%$ ao grau 3 ; e apenas $4,2 \%$ ao grau $4^{(12)}$. Um estudo realizado em Portugal indicou que as flebites graus $1(37,0 \%)$ e $2(53,6 \%)$ também foram as mais presentes ${ }^{(24)}$. Identificar a flebite nos primeiros graus previne a ocorrência de danos permanentes e temporários para o paciente.

Os medicamentos podem contribuir para o desenvolvimento de flebite em razão das propriedades químicas, $\mathrm{pH}$ e osmolaridade ${ }^{(23)}$. Os antibióticos estão relacionados aos riscos para o desenvolvimento de flebite ${ }^{(25)}$ e risco maior de apresentar esse EA em grau mais avançado ${ }^{(14)}$. Os antibióticos e analgésicos são fármacos causadores de flebite devido aos aspectos relativos à diluição e às interações medicamentosas, isso quando a salinização ao término da infusão não é realizada ${ }^{(23)}$.

The Infusion Nursing Standards of Practice recomenda o uso de cateter com sistema de estabilização integrada, sempre que possível, visto diminuir o risco de flebite ${ }^{(2)}$. A escolha do dispositivo é fundamental, antes da punção do AVP é preciso avaliar os pacientes e conhecer as propriedades do fármaco que será infundido, como no caso de medicamentos irritantes e vesicantes.

Ressalta-se que a ocorrência de flebite eleva os custos assistenciais associados ao estabelecimento de um novo AVP, além dos cuidados que a tal EA demandam, aumentando o consumo de materiais e o tempo dispendido pelos recursos humanos. Como exemplo, estudo realizado nos Estados Unidos da América indicou que a ocorrência de 566 flebites, no período de um mês, resultou na necessidade de 396 novas punções, além disso, o custo estimado com o novo AVP correspondeu a US\$13.860,00 $20^{(26)}$.

As notificações analisadas indicaram que os pacientes estavam, na sua maioria, distantes do posto de enfermagem. Portanto, o período que a equipe demanda entre o atendimento do chamado e o deslocamento até o quarto do paciente pode ocasionar piora da lesão no vaso, devido ao tempo em pausar/interromper a medicação e avaliar o dispositivo.

Um aspecto limitante do presente estudo decorreu de que $20,45 \%$ das notificações não possuíam registros sobre os procedimentos adotados na vigência da flebite. Ressalta-se que a notificação correta é de extrema importância para a quantificação da ocorrência da flebite e realização da análise das causas e fatores contribuintes, assim visando à avaliação de riscos e à adoção de medidas para se melhorar a segurança do paciente ${ }^{(10)}$. Nessa lógica, os profissionais médicos e de enfermagem devem registrar no prontuário as informações relativas ao cuidado prestado de forma clara, legível, objetiva, completa, com data, hora, assinatura e número do seu registro profissional ${ }^{(27-28)}$.

Frente à necessidade de identificar precocemente os sinais/sintomas de flebite, avaliar e documentar o grau de flebite na prática de enfermagem, a utilização de uma escala adequada torna-se imprescindível e poderá auxiliar o enfermeiro na tomada de decisão e no estabelecimento do plano de cuidados ${ }^{(29)}$.

A orientação sistemática dos pacientes e familiares, como uma estratégia adotada no hospital campo de estudo, é importante fonte de informação e prevenção de EA. Além disso, o hospital dispõe de um protocolo específico para AVP que diminuiu o desconforto para o paciente devido às múltiplas punções e reduz o custo relacionado ao uso de materiais e recursos humanos nas tentativas de punções sem sucesso.

Por fim, entende-se que analisar as causas e os motivos associados a ocorrência do EA flebite contribui com o aprimoramento do conhecimento sobre o tema, além de fornecer subsídios para a construção de protocolos assistenciais e intervenções educativas junto aos profissionais de saúde, privilegiando a adoção de medidas preventivas. 


\section{CONCLUSÃO}

Por fim, foram notificadas 107 flebites relativas a 96 pacientes, a maioria com registro da ocorrência de uma flebite (91,7\%), do sexo masculino $(53,1 \%)$, na faixa etária de 60-69 anos $(23,0 \%)$ e com tempo de permanência inferior a quatro dias $(30,2 \%)$.

O enfermeiro foi o profissional que realizou a maioria das notificações $(68,2 \%)$. Além disso, houve predomínio: da ocorrência de flebite nos dispositivos com tempo inferior a $24 \mathrm{~h}(38,3 \%)$, da classificação em grau $2(45,8 \%)$, da infusão de antibióticos $(46,7 \%)$, da localização do leito distante do posto de enfermagem $(52,3 \%)$ e da presença de acompanhante $(82,2 \%)$. A classificação de dano causado pela flebite indicou que $93,5 \%$ dos pacientes sofreram danos leves; $4,7 \%$, danos moderados; $1,9 \%$ não sofreram danos. Não houve registro de pacientes classificados como dano grave ou óbito.

\section{RESUMO}

Objetivo: Analisar as causas e os motivos associados à ocorrência de flebite na Unidade de Internação Clínica de um hospital geral, que é de grande porte e privado. Método: Pesquisa quantitativa, exploratório-descritiva, retrospectiva e documental, realizada a partir da consulta aos formulários eletrônicos de notificação da ocorrência de flebite referentes ao ano de 2017. Resultados: Foram notificadas 107 flebites relativas a 96 pacientes, a maioria (91,7\%) com uma flebite, do sexo masculino (53,1\%), na faixa etária de 60-69 anos $(23,0 \%)$ e com tempo de permanência inferior a quatro dias (30,2\%). A maioria (68,2\%) das notificações foi realizada por enfermeiros, tendo predominado a ocorrência de flebite nos dispositivos com tempo inferior a $24 \mathrm{~h}(38,3 \%)$, a classificação flebite grau 2 (45,8\%), a infusão de antibióticos (46,7\%), a localização do leito distante do posto de enfermagem $(52,3 \%)$ e a presença de acompanhante (82,2\%). A classificação de danos indicou que $93,5 \%$ dos pacientes sofreram danos leves, $4,7 \%$, danos moderados e $1,9 \%$ não sofreram danos Conclusão: Conhecer as causas e os motivos associados à ocorrência de flebite poderá subsidiar os processos decisórios, gerencial e assistencial, quanto aos investimentos em estratégias preventivas ou de mitigação de riscos.

\section{DESCRITORES}

Pacientes Internados; Cateterismo Periférico; Flebite; Cuidados de Enfermagem; Unidades Hospitalares.

\section{RESUMEN}

Objetivo: Analizar las causas y los motivos asociados a la aparición de flebitis en la Unidad de Hospitalización Clínica de un hospital general privado y de gran envergadura. Método: Se trata de una investigación cuantitativa, exploratoria descriptiva, retrospectiva y documental, realizada mediante la consulta de formularios electrónicos de notificación del acaecimiento de flebitis en 2017. Resultados: Se reportaron 107 flebitis relativas a 96 pacientes, la mayoría de ellos (91,7\%) con una flebitis, del sexo masculino (53,1\%), en el grupo de edad de 60-69 años (23,0\%) y con estancia inferior a cuatro días (30,2\%). El personal de enfermería realizó la mayoría de las notificaciones $(68,2 \%)$, con predominio de aparición de flebitis en los dispositivos con tiempo inferior a $24 \mathrm{~h}$ (38,3\%), la clasificación de la flebitis en grado 2 (45,8\%), la infusión de antibióticos (46,7\%), la ubicación de la cama lejos del puesto de enfermería (52,3\%) y la presencia de un acompañante (82,2\%). La clasificación de los daños indicó que el 93,5\% de los pacientes sufría daños leves, el 4,7\%, daños moderados y el $1,9 \%$ no había sufrido daños. Conclusión: Conocer las causas y los motivos asociados a la aparición de flebitis puede subsidiar los procesos decisorios, gerenciales y asistenciales, en relación con las inversiones en estrategias preventivas o de mitigación de riesgos.

\section{DESCRIPTORES}

Pacientes Internos; Cateterismo Periférico; Flebitis; Atención de Enfermería; Unidades Hospitalarias.

\section{REFERÊNCIAS}

1. Zheng GH, Yang L, Chen HY, Chu JF, Mei L. Aloe vera for prevention and treatment of infusion phlebitis. Cochrane Database Syst Rev. 2014;(6):CD009162. https://doi.org/10.1002/14651858.CD009162.pub2

2. Gorski LA. The 2016 Infusion therapy standards of practice. Home Healthc Now. 2017;35(1):10-8. https://doi.org/10.1097/ NHH.0000000000000481

3. Abdul-Hak CK, Barros AF. The incidence of phlebitis in a Medical Clinical Unit. Texto Contexto Enferm. 2014;23(3):6338. https://doi.org/10.1590/0104-07072014000900013

4. Cicolini G, Manzoli L, Simonetti V, Flacco ME, Comparcini D, Capasso L, et al. Phlebitis risk varies by peripheral venous catheter site and increases after 96 hours: a large multi-centre prospective study. J Adv Nurs. 2014;70(11):2539-49. https://doi.org/10.1111/jan.12403

5. Kohn LT, Corrigan JM, Donaldson MC, editors. To err human: building a safer health system. Washington: National Academy Press; 1999 .

6. World Health Organization. World alliance for patient safety: forward programme [Internet]. Geneve: World Health Organization; 2004 [cited 2017 Sep 15]. Available from: http://www.who.int/patientsafety/en/brochure_final.pdf

7. Brasil. Ministério da Saúde. Programa Nacional de Segurança do Paciente [Internet]. Brasília, DF: Ministério da Saúde; 20172004 [cited 2017 Sep 16 Available from: http://portalsaude.saude.gov.br/index.php/o-ministerio/principal/secretarias/sas/dahu/seguranca-do-paciente

8. Brasil. Ministério da Saúde. Portaria No 529, de 1 de abril de 2013. Institui o Programa Nacional de Segurança do Paciente (PNSP). Brasília, DF; 2013 [cited 2017 Sep 16]. Available from: http://bvsms.saude.gov.br/bvs/saudelegis/gm/2013/prt0529_01_04_2013.html

9. World Health Organization. Global action on patient safety [Internet]. 2019 [cited 2020 Feb 4] May 28. Available from: https://apps.who. int/gb/ebwha/pdf_files/WHA72/A72_R6-en.pdf

10. Urbanetto JS, Peixoto CG, May TA. Incidence of phlebitis associated with the use of peripheral IV catheter and following catheter removal. Rev Latino-Am Enferm. 2016;24:e2746. http://dx.doi.org/10.1590/1518-8345.0604.2746

11. Beccaria LM, Machado BD, Bertolli ES, Contrin LM, Werneck AL. Incidente of phlebitis in adult patients. Rev Enferm UFPE On Line. 2018;12(3):745-52. https://doi.org/10.5205/1981-8963-v12i3a230454p745-752-2018 
12. Tertuliano AC, Borges JLS, Fortunato RAS, Poveda VB, Oliveira AL. [Phlebitis associated with peripheral intravenous catheter among inpatients of a Hospital in Vale do Paraíba]. Rev Min Enferm. 2014;18(2):334-45. Portuguese. https://doi.org/10.5935/1415-2762.20140026

13. Souza AE, Oliveira JL, Dias DC, Nicola AL. [Prevalence of phlebitis in adult patients admitted to a university hospital]. Rev RENE. 2015;16(1):114-22. Portuguese. https://doi.org/10.15253/2175-6783.2015000100015

14. Nyika ML, Mukona D, Zvinavashe M. Factors contributing to phlebitis among adult patients admitted in the medical-surgical units of a Central Hospital in Harare, Zimbabwe. J Infus Nurs. 2018;41(2):96-102. https://doi.org/10.1097/NAN.0000000000000265

15. Milutinović D, Simin D, Zec D. Risk factor for phlebitis: a questionnaire study of nurses' perception. Rev Latino-Am Enferm. 2015;23(4):67784. https://doi.org/10.1590/0104-1169.0192.2603

16. Danski MT, Johann DA, Vayego SA, Oliveira GR, Lind J. Complications related to the use of peripheral venous catheters: a randomized clinical tria. Acta Paul Enferm. 2016;29(1):84-92. https://doi.org/10.1590/1982-0194201600012

17. Braga LM, Parreira PM, Oliveira AS, Mónico LS, Arreguy-Sena C, Henriques MA. Phlebitis and infiltration: vascular trauma associated with the peripheral venous catheter. Rev Latino-Am. Enferm. 2018;26:e3002. https://doi.org/10.1590/1518-8345.2377.3002

18. Enes SM, Opitz SP, Faro AR, Pedreira ML. Phlebitis associated with peripheral intravenous catheters in adults admitted to hospital in the Western Brazilian Amazon. Rev Esc Enferm USP. 2016;50(2):263-71. https://doi.org/10.1590/S0080-623420160000200012

19. Furini AC, Nunes AA, Dallora ME. Notifications of adverse events: characterization of the events that occurred in a hospital complex. Rev Gaúcha Enferm. 2019;40(esp):e20180317. https://doi.org/10.1590/1983-1447.2019.20180317

20. Brasil. Ministério da Saúde. Agência Nacional de Vigilância Sanitária. Relatório Brasil e Estado de São Paulo - 2018. Brasília, DF: Anvisa; 2018.

21. Pasalioglu KB, Kaya H. Catheter indwell time and phlebitis development during peripheral intravenous catheter administration. Pak J Med Sci. 2014;30(4):725-30. https://doi.org/10.12669/pjms.304.5067

22. Rickard CM, Webster J, Wallis MC, Marsh N, McGrail MR, French V, et al. Routine versus clinically indicated replacement of peripheral intravenous catheters: a randomised controlled equivalence trial. Lancet. 2012;380(9847):1066-74. https://doi.org/10.1016/S0140-6736(12)61082-4

23. Oliveira ECS, Oliveira APB, Oliveira RC. Characterization of phlebitis notifications to risk management in hospital sentinel network. Rev Baiana Enferm. 2016;30(2):1-9. Portuguese https://doi.org/10.18471/rbe.v30i2.15361

24. Salgueiro-Oliveira A, Parreira P, Veiga P. Incidence of phlebitis in patients with peripheral intravenous catheters: the influence of some risk factors. Aust J Adv Nurs [Internet]. 2012 [cited 2019 Mar 16;30(2):32-9. Available from: https://search.informit.com.au/documentSu mmary; dn=089105424571588; res=ielapa

25. Urbanetto JS, Freitas AP, Oliveira AP, Santos JC, Muniz FO, Silva RM, et al. Risk factors for the development of phlebitis: an integrative review of literature. Rev Gaúcha Enferm. 2018;38(4):e57489. https://doi.org/10.1590/1983-1447.2017.04.57489

26. Jones RK. Short peripheral catheter quality and economics: the intravenous quotient. J Infus Nurs. 2018;41(6):36571. https://doi.org/10.1097/NAN.0000000000000303

27. Conselho Federal de Medicina. Resolução CFM nº 193/2009. Código de Ética Médica. Capítulo X - Documentos médicos. Diário Oficial União, Brasília, DF. 2009 Sep 17.

28. Conselho Federal de Enfermagem. Resolução COFEN nº 311/2007. Código de Ética dos Profissionais de Enfermagem. Diário Oficial União, Brasília, DF. 2007 Feb 13.

29. Braga LM, Salgueiro ASO, Pereira HMA, Alves RM, Vidal RCJ, Pereira SAG et al. Translation and adaptation of the Phlebitis Scale for the Portuguese population. Rev Enf Ref. 2016;serIV(11):101-9. . https://doi.org/10.12707/RIV16048 